\title{
Tungiasis: A Rare Parasitic Infestation in Genitals of a Native Male from Kathmandu
}

\author{
Shreya Shrivastav, ${ }^{1}$ Ram Chandra Adhikari ${ }^{1}$ \\ ${ }^{1}$ Institute of Medicine, Trbhuvan University Teaching Hospital Maharajgunj Kathmandu, Nepal.
}

\begin{abstract}
Tungiasis is a common parasitic infection inmany parts of the world, including South and Central America and parts of Africa. It is rare in other places, such as in India and Pakistan and imported cases have been reported in Taiwan, Italy, the United States of America, etc. Our diagnosis was made based on histopathologiocal features of the parasite, which corresponds too ther reports and description of tungiasis. ${ }^{1-3}$ It was treated by surgical excision alone. The fact that the disease has never been reported in Nepal makes it difficult to diagnose clinically, but should be considered in the future. Histopathology can aid in diagnosingthis rare condition.

Keywords: Nepal; tunga penetrans; tungiasis.
\end{abstract}

\section{INTRODUCTION}

Tungiasis is a cutaneous parasitic infection caused by the parasite, Tunga penetrans ( $T$. penetrans). The common name is sand flea and it is endemic in South America. It is also common in eastern and southern Africa. It has rarely been reported in the South Asia, including in India and Pakistan, ${ }^{4}$ but not in Nepal, to this date. Most commonly it affects the feet, mainly the inter digital and subungal regions. Other parts of the body such as the buttocks and genital region can also be affected.
A 65 year old native man of Kathmandu, Nepal presented to the surgical OPD with a painful small nodule on the shaft of penis for the past 15 days. The patient had no history of trauma, no travel history and no history of contact with any animals. He was not a known case of any sexually transmitted infection and no investigations along the line of syphilis was done.

On examination, the nodule was about $3-4 \mathrm{~mm}$ in size and was clinically thought to be a papilloma. The lesion was excised and sent for histopathological evaluation.

\section{CASE REPORT}

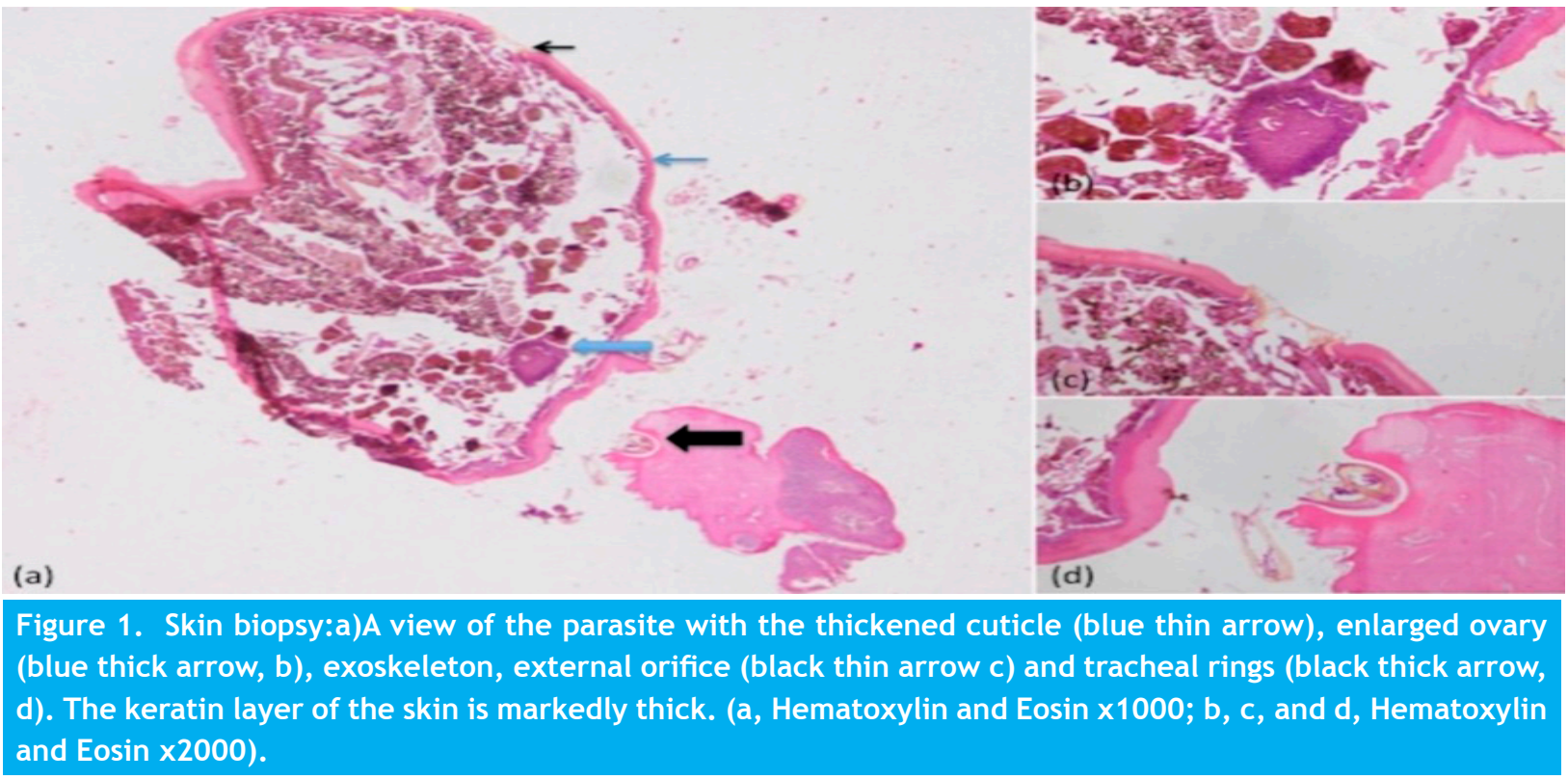

DOI: http://dx.doi. org/10.3126/jnhrc.v15i3.18860
Correspondence: Shreya Shrivastav, Institute of Medicine, Tribhuvan University Teaching Hospital Maharajguni Kathmandu, Nepal. Email: shreya_shrivastav@ hotmail.com, shreya_shrivastav@iom.edu.np, Phone : +9779860479932. 
Grossly, the specimen consisted of a grey to black, skin covered tissue. A Hematoxylin and Eosin section revealed a tiny bit of stratified squamous epithelium with marked hyperkeratosis. Along with it, there was a parasite with a thick cuticle, exoskeleton, external orifice of the organism, tracheal rings and an enlarged ovary (Figure 1). The differential diagnoses considered were scabies and tungiasis.

Although scabies would have been the more probable diagnosis based on the geographical and sociocultural factors, the morphology in our case did not match that of the Sarcoptesscabiei. Based on morphology, a diagnosis of tungiasis was made.

No further treatment was undertaken and the patient has been asymptomatic since then.

\section{DISCUSSION}

Tungiasis is a skin infection caused by the sand flea, $T$. penetrans. It is also known by several other names such as chigoe flea, sand flea, chigo, chica, jigger, pico, pique, and chique. ${ }^{1-3}$ The parasite was first reported by Gonzalez Fernandez de Oviedo y Valdes in 1525 in Haiti. ${ }^{5}$ The parasite is endemic in South America with a prevalence of $34 \%$ to $51 \%$ in various communities in Brazil. ${ }^{6}$ In 1872, the parasite is said to have travelled to Angola in Africa on a trade ship. Since then, the parasite became common in certain parts of Africa, such as Uganda and Nigeria. ${ }^{5-6}$

It is from Africa, through various military movements during the British rule, that the parasite spread to Pakistan and India. ${ }^{4}$ However, the cases reported in these countries are rare. It is also seen in tourists and other workers who return to their home countries after travelling to endemic regions. Nineteen imported cases have been reported from Italy. ${ }^{7}$ There have been no case reports on tungiasis from Nepal.

The parasite is found in warm, dry sand and most commonly affects people walking barefoot in the sand. In endemic regions, it is especially common in poor communities that cannot afford good footwear and walk barefoot in the sand. The parasite has also been found in other parts of the body, such as hands, breast, back, buttocks, thighs and legs. ${ }^{7} \mathrm{~A}$ rare case of the parasite is reported in the oral cavity. ${ }^{8}$ Other than humans, tungiasis is also known to affect dogs, cats, rats and various other mammals.

The tunga larva is a free living organism and lives in the soil. It measures about $1 \mathrm{~mm}$ in diameter. The life cycle lasts for one month. The gravid female flea penetrates the skin and stays in the epidermis. It is believed to secrete keratinolytic substances that aid this process. Here, it enlarges to a size of about $1 \mathrm{~cm}$. It maintains an external orifice through which it communicates outside the skin. It excretes faeces and eggs outside by means of this orifice. After this process, which takes about two weeks, the parasite dies and is sloughed off.

The disease presents as a single or sometimes multiple grey to whitish nodules with a central black opening in the center, which corresponds to the external opening of the organism. Nodules are usually painless but may sometimes be painful, especially as the organism enlarges. It normally resolves on its own. In endemic areas, locals recognize these lesions easily and treat it themselves, seeking medical attention only when there are complications. However, in imported cases or in places where the disease is rare or not known to occur, excision and histopathological examination is required for diagnosis. Not uncommonly, there are complications such as bacterial superinfections, tetanus, septic ulcers, abscesses, cellulitis, gangrene, thrombophlebitis or autoamputation of toes. As many as $25 \%$ cases could get secondary infection. ${ }^{7}$

As far as treatment is concerned, in endemic areas, patients remove the lesions themselves or by family members by means of needles, knives, etc. This is accompanied by the use of various disinfectants like mercurochrome, ethanol and potassium permanganate and other oily preparations and ointments when there are numerous sand flies. When patients do seek medical treatment, the first choice of therapy is complete surgical removal, often with a hypodermic needle along with topical application of ivermectin, topical thiabendazole or topical metrifonate, which have been found to significantly reduce the number of lesions. ${ }^{9}$ In addition, tetanus prophylaxis is recommended or even mandatory. ${ }^{10}$ In endemic areas, use of protective footwear is recommended for prophylaxis. In endemic countries as well as in our context, a general need for hygiene and better living conditions cannot be overemphasized.

\section{CONCLUSIONS}

Tungiasis should be considered in the differential diagnosis of lesions in the foot, buttocks, genitalia, arms and legs, even in our parts of the world and histopathology can confirm the diagnosis. 


\section{REFERENCES}

1. Pampiglione S, Fioravanti ML, Gustinelli A, Onore G, Mantovani B, Luchetti A, et al.Sand flea (Tunga spp.) infectionsin humans and domestic animals: state of the art. Med Vet Entomol. 2009;3:172-86.[Pub Med]

2. Macías PC, Sashida PM. Cutaneous infestation by Tungapenetrans. Int J Dermatol. 2000;39:296-8.

3. Feldmeier H, Eisele $M$, Van Marck E, Mehlhorn H, Ribeiro R, Heukelbach J. Investigations on the biology, epidemiology, pathology and control of Tungapenetrans in Brazil IV. Clinical and histopathology. Parasitol Res. 2004;94:275-82. [Pub Med]

4. Sane S Y, Satoskar R R. Tungiasis in Maharashtra (a case report). J Postgrad Med. 1985;31:121.[Full Text]

5. Hoeppli R. Early references to the occurrence of Tungapenetrans inTropical Africa. Acta Trop. 1963;20:14353.[Full Text]
6. Wilcke T, Heukelbach J, Cesar Saboia MR, Kerr-Pontes RL, Feldmeier H (2002) High prevalence of tungiasis in a poor neighbourhood in Fortaleza, Northeast Brazil. Acta Trop. 83:255-8

7. Veraldi S, Valsecchi M, Imported tungiasis: a report of 19 cases and review of the literature. Int J Dermatol. 2007 Oct;46(10):1061-6. [Full Text]

8. Sentongo and Wabinga Tungiasis presenting as a soft tissue oral lesion. BMC Oral Health. 2014;14-112. [Pub Med]

9. Heukelbach J, Eisele M, Jackson A, Feldmeier H Topical treatment of tungiasis: a randomized, controlled trial. Ann Trop Med Parasitol. 2003;97:743-9.[Full Text]

10. Bell A, Neely CL, Peeples J Tungiasis in Tennessee. South Med J. 1979;72:141-3. [Pub Med] 